\title{
Atypical haemolytic uraemic syndrome as a complication of induction chemotherapy for acute lymphoblastic leukaemia
}

\author{
D Chandra, S Lawson, P Ramani
}

J Clin Pathol 2004;57:667-669. doi: 10.1136/icp.2003.013979

This report describes a case of fatal haemolytic uraemic syndrome (HUS) developing in a child with acute lymphoblastic leukaemia (ALL) during induction chemotherapy. The aetiology in this case is uncertain but it may have resulted from treatment with L-asparaginase or vincristine. The possibility of HUS during induction chemotherapy for ALL should be considered early on in the treatment regimen, if clinical signs and symptoms suggest this diagnosis, so that appropriate treatment can be instituted.

W e report a fatal case of haemolytic uraemic syndrome (HUS) developing in a child with acute lymphoblastic leukaemia (ALL) during induction chemotherapy. The aetiology was uncertain but treatment with L-asparaginase or vincristine might be involved. The possibility of HUS during induction chemotherapy for ALL should be considered early on, if clinical signs and symptoms suggest this diagnosis, so that appropriate treatment can be instituted.

\section{CASE REPORT}

A four year old girl presented with a five day history of lethargy and bruising. A diagnosis of common acute lymphoblastic leukaemia (ALL) was made after appropriate investigations. The patient was started on induction chemotherapy according to schedule A of the Medical Research Council UK ALL 97/01 protocol. She received dexamethasone $6.5 \mathrm{mg} / \mathrm{m}^{2} /$ day, vincristine $1.5 \mathrm{mg} / \mathrm{m}^{2} /$ week, and L-asparaginase (Escherichia coli) (Elspar; Merck, West Point, USA) 6000 units $/ \mathrm{m}^{2}$ on alternate days. She was discharged eight days after diagnosis with further treatment on an outpatient basis.

She was readmitted 10 days after her first admission (day 16 of chemotherapy), with fever, neutropenia, and cellulitis over the left gluteal region.

She was treated with oxygen, intravenous fluids, meropenem, gentamicin, flucloxacillin, and fluconazole and continued to receive induction chemotherapy.

The initial blood culture grew $E$ coli, which was not the O157 strain and was negative for verocytotoxin. It was sensitive to meropenem, gentamicin, and ceftazidime. The patient's fever subsided within 24 hours of starting antibiotics.

Four days (day 20 of chemotherapy) after readmission the patient developed deranged renal function with declining urine output. Biochemical investigations (previously normal) showed a rising blood urea and serum creatinine. An abdominal ultrasound revealed an increase in size and echogenicity of both kidneys. She also had an increased blood and platelet requirement and had poor platelet increments after transfusions. Figure 1 shows the results of the haematological and biochemical investigations.
A possibility of drug induced interstitial nephritis resulting in acute renal failure was considered and meropenem and gentamicin were replaced with ceftazidime. Her fluid intake was restricted to $400 \mathrm{ml} / \mathrm{m}^{2} /$ day and she was investigated for platelet refractoriness.

Over the next two days, the patient became increasingly drowsy (Glasgow coma scale (GCS) 13/15) and her renal function and urine output deteriorated further. Investigations revealed raised lactate dehydrogenase (6353 IU/ litre; normal range, 470-900), reduced serum haptoglobin $(<2.43 \mathrm{gm} /$ litre; normal range, 4.5-20.5), negative direct Coombs test, and absence of antiplatelet and anti-HLA antibodies. The blood film showed red cell fragmentation and the clotting screen, including fibrinogen and D-dimer values, was normal. A bone marrow aspirate showed morphological remission from ALL. Repeat blood and stool cultures were negative.

The patient was started on haemodialysis but this had to be discontinued after a few hours because she developed focal convulsions. A cranial computed tomography (CT) scan revealed no abnormalities and gradual haemofiltration was carried out without further complication.

The diagnosis of haemolytic uraemic syndrome (HUS) was contemplated at this stage and a renal biopsy was performed. The renal biopsy revealed thickened glomerular capillary walls and focal mesangiolysis. There was tubular dilatation, and many of the tubules contained proteinaceous and cellular casts, indicating acute tubular necrosis (fig 2). Immunoperoxidase staining showed IgM deposition in the mesangium. Electron microscopy of the renal biopsy confirmed HUS-like changes, with subendothelial deposition of electron dense strands and acellular "fluff". The diagnosis of HUS was confirmed.

In view of the diagnosis, the patient underwent a single volume plasma exchange, but had a sudden neurological deterioration (GCS 6/15) and haemodynamic collapse. A CT scan of the head revealed a large left frontoparietal intracerebral haemorrhage, which was surgically evacuated. There was no neurological improvement after evacuation and she remained comatose (GCS 4/15) with a left hemiparesis. Her renal function showed some improvement after daily double volume plasma exchange and haemofiltration.

Two days later, she had further extensive intracerebral bleeding, confirmed on CT scan. The patient died shortly after active treatment was withdrawn following discussion with the parents.

\section{DISCUSSION}

Thrombotic microangiopathy manifesting as HUS or thrombotic thrombocytopenic purpura (TTP) is a common complication in patients with cancer. HUS and TTP probably

Abbreviations: ALL, acute lymphoblastic leukaemia; $\mathrm{CT}$, computed tomography; HUS, haemolytic uraemic syndrome; TTP, thrombotic thrombocytopenic purpura 


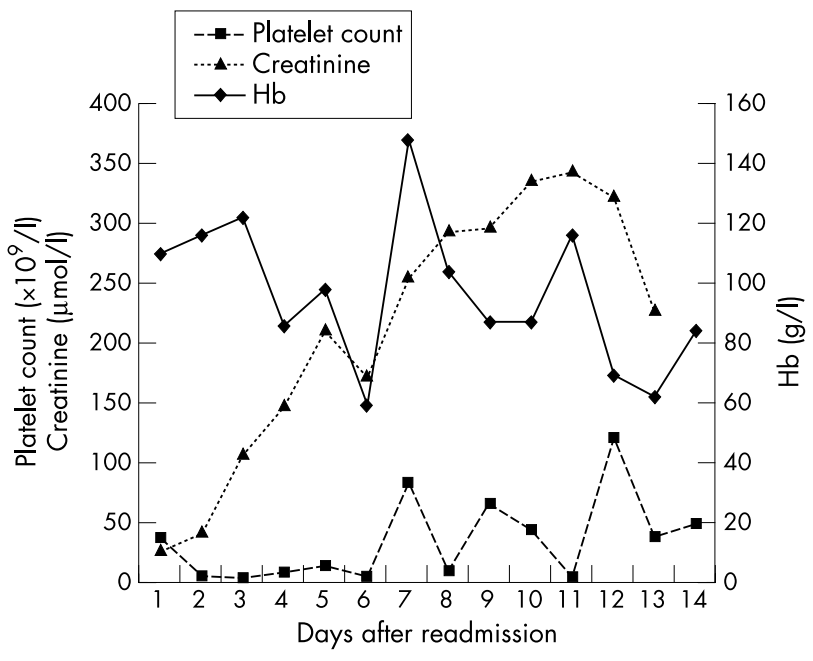

Figure 1 Haemoglobin, platelet count, and serum creatinine values after readmission.

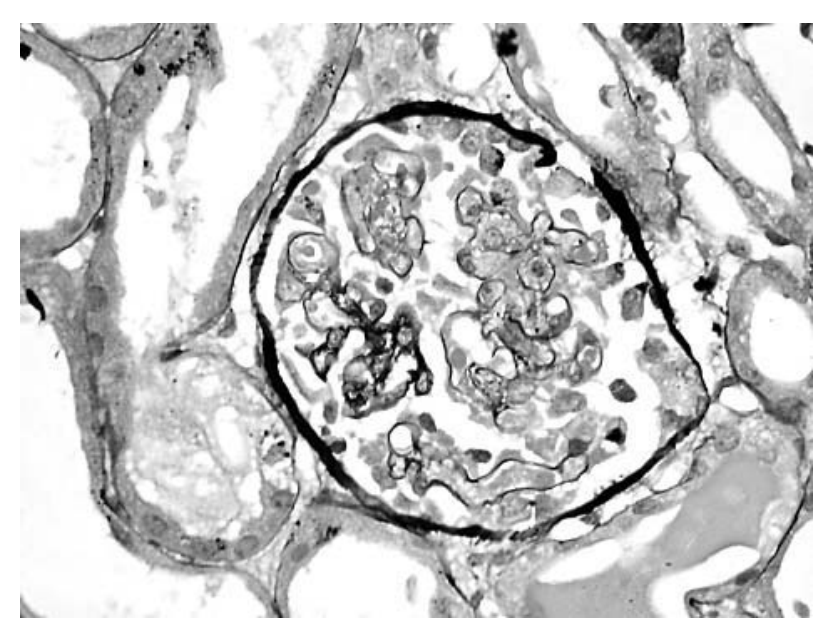

Figure 2 Histological examination of right kidney biopsy. The glomerulus shows pronounced congestion, filling of capillary lumens by partly fragmented red blood cells, and focal mesangiolysis. A capsular reaction is seen on the right. Haematoxylin and eosin stain; original magnification, $\times 260$.

represent different ends of the same disease continuum. HUS is characterised by microangiopathic haemolysis, thrombocytopenia, and acute renal failure and, unlike TTP, is not associated with severe deficiency of von Willebrand factor metalloprotease. Typical HUS often follows a prodromal diarrhoeal illness, often as a result of $E$ coli or other verocytotoxin producing enteropathogens. Atypical HUS has a more heterogeneous aetiology and has been associated with cancer, chemotherapeutic agents, bone marrow transplantation, and viral infections including varicella, echovirus, coxsackie A and B, and human immunodeficiency virus, in addition to neuraminidase producing Streptococcus pneumoniae.

Our patient had microangiopathic haemolysis, thrombocytopenia, and acute renal failure. The clinical course was complicated by neurological abnormalities, which highlights the considerable overlap in the clinical features and pathophysiology of HUS and TTP and the temptation to consider them as a single disease entity. The only other differential diagnosis was disseminated intravascular coagulopathy, which in the presence of a normal coagulation screen was unlikely. The renal biopsy confirmed the diagnosis of HUS, which in the absence of diarrhoeal illness and the
Take home messages

- This report describes a case of fatal haemolytic uraemic syndrome (HUS) during induction chemotherapy for acute lymphoblastic leukaemia (ALL)

- Although the aetiology is unclear, it may have resulted from treatment with $\mathrm{L}$-asparaginase or vincristine

- If clinical signs and symptoms are suggestive of HUS during induction chemotherapy for ALL this diagnosis should be considered early on, so that appropriate treatment can be instituted

isolation of an enteropathogen from stool culture was of atypical aetiology.

Among the cancers associated with HUS or TTP, gastric adenocarcinoma predominates, followed by breast cancer, colorectal cancer, and cancer of unknown primary origin. ${ }^{12}$ There have been very few reports of HUS in patients with acute leukaemia who have not undergone bone marrow transplantation. In three previous case reports, HUS preceded the onset of ALL. Salcedo and Fusner, ${ }^{3}$ Martini et al, ${ }^{4}$ and Hahn and colleagues ${ }^{5}$ have reported patients who presented with atypical HUS eight, one, and five months, respectively, before being diagnosed with ALL. The authors have suggested that HUS may be an uncommon clinical presentation of ALL.

Chemotherapeutic drugs often implicated in the aetiology are mitomycin $\mathrm{C}$, cyclosporin, tamoxifen, bleomycin, and daunorubicin. ${ }^{5}$

"We suspect that this patient developed haemolytic uraemic syndrome as a complication of induction chemotherapy for acute lymphoblastic leukaemia"

As part of induction chemotherapy our patient was treated with vincristine, L-asparaginase ( $E$ coli), and dexamethasone. None of these drugs has been shown to cause HUS. There is only one previous report where a patient developed HUS while receiving chemotherapy for ALL. Kanchi et al reported a child with ALL who developed recurrent HUS as a possible complication of treatment with vincristine. ${ }^{6}$ Paradoxically, vincristine and steroids have been used to treat patients with refractory TTP. ${ }^{78}$ This patient also received meropenem, gentamicin, ceftazidime, flucloxacillin, and fluconazole. None of these drugs has previously been reported to cause HUS.

Treatment modalities for cancer associated HUS are similar to those used in typical HUS, with appropriate management of fluid and electrolytes, dialysis support, and plasma infusion or exchange.

Despite extensive investigations all the possible causes of HUS could not be ruled out in our patient. We suspect that this patient developed HUS as a complication of induction chemotherapy for ALL. Because vincristine and steroids are used to treat refractory TTP, a possible aetiological agent in our patient is $E$ coli derived L-asparaginase, which is known to predispose patients to thrombosis by reducing antithrombin and protein $\mathrm{C}$ concentrations, but has not previously been reported to cause HUS or TTP.

It is our view that the possibility of HUS as a complication during ALL chemotherapy should be considered early, if the clinical picture suggests such a diagnosis, so that appropriate treatment can be instituted without delay.

\section{ACKNOWLEDGEMENTS}

We thank Mr D Redfern for the electronic microphotograph. 


\section{Authors' affiliations}

D Chandra, S Lawson, P Ramani, Birmingham Children's Hospital, Steelhouse Lane, Birmingham B4 6NH, UK

Correspondence to: $\operatorname{Dr} D$ Chandra, Department of Paediatric Haematology, Birmingham Children's Hospital, Steelhouse Lane, Birmingham, B4 6NH, UK; deepak.chandra@bch.nhs.uk

Accepted for publication 28 November 2003

\section{REFERENCES}

1 Gordon LI, Kwaan HC. Thrombotic microangiopathy manifesting as thrombotic thrombocytopenic purpura/haemolytic uremic syndrome in the cancer patient. Semin Thromb Hemost 1999;25:217-21.
2 Kwaan HC, Gordon LI. Thrombotic microangiopathy in the cancer patient. Acta Haematol 2001;106:52-6.

3 Salcedo JR, Fusner J. Hemolytic uremic syndrome followed by acute lymphocytic leukaemia. Int J Pediatr Nephrol 1986;7:169-72.

4 Martini G, Dall'Amico R, Murer L, et al. Haemolytic-uremic syndrome as a presenting form of acute lymphocytic leukaemia. Ann Hematol 2000;79:452-4.

5 Hahn H, Ha IS, Choi HS, et al. Acute leukaemia: an association with atypical hemolytic uremic syndrome. Pediatr Nephrol 2003;18:703-5.

6 Kanchi H, Webb NJA, Eden OB. Hemolytic uremic syndrome secondary to the treatment of acute lymphoblastic leukaemia. J Pediatr Hematol Oncol 2000;22:483-4.

7 Bell WR, Braine HG Ness PM, et al. Improved survival in thrombotic thrombocytopenic purpura-hemolytic uremic syndrome: clinical experience in 108 patients. N Engl J Med 1991;325:398-403.

8 Gutterman LA, Stevenson TD. Treatment of thrombotic thrombocytopenic purpura with vincristine. JAMA 1982;247:1433-6. 